\title{
Investigating Student Learning with Accessible Interactive Physics Simulations
}

\author{
Elise C. Morgan, Emily B. Moore \\ Department of Physics, University of Colorado Boulder, UCB 390, Boulder, Colorado, 80309
}

\begin{abstract}
The PhET Interactive Simulations project has begun an initiative to increase the accessibility of its suite of science simulations. In this work, we focus on use of the PhET sim Capacitor Lab: Basics by two visually impaired learners. Comparing responses to pre and posttest questions about capacitance and circuits, our results indicate that after using the simulation with new accessibility features, both learners better understood the relationship between plate separation, area, and capacitance. Additionally, while only one learner connected the charged capacitor to the light bulb in the simulation during use, both answered that the light bulb will be illuminated in posttest questions. These findings indicate that visually impaired students can master the learning goals of a PhET sim with well-designed accessibility features. Findings from this research contribute to understanding how to develop physics education resources capable of supporting diverse students, including students with disabilities.
\end{abstract}

Keywords: Simulation, screen reader, accessibility, circuit, capacitor, auditory descriptions, keyboard navigation PACS: 01.40.Fk, 01.50.H-

\section{INTRODUCTION}

The $\mathrm{PhET}$ Interactive Simulations project at the University of Colorado Boulder (http://phet.colorado.edu) has published over 150 science and mathematics simulations (sims). These sims are used over 100 million times per year around the world [1]. With a reliance on mouse or touch input and visual outputs, PhET sims are currently inaccessible to many students with disabilities, including students with motor impairments needing alternate input methods or visually impaired students needing non-visual access to the sim environment. In order to better support students with disabilities, PhET has begun an initiative to better understand the needs of students with disabilities in order to broaden the accessibility of the sims. Here we focus on research conducted to understand how accessibility features in one PhET sim, Capacitor Lab: Basics, can support the learning of visually impaired students.

There exists a significant achievement gap between students with and without disabilities in science [2,3]. While the vast majority of students with disabilities are taught in general education classes, these students are put at a disadvantage when the class uses educational resources that are highly visual and not accessible, including essentially all interactive simulations. In these situations, students with visual impairments are either left to observe as their peers engage with the learning resources, or are provided with a less interactive activity to complete instead. In contrast, students with disabilities who participate in active learning activities and engage with their peers are more likely to experience positive learning outcomes and progress at their instructional level [4].

Recent efforts by the accessibility community have resulted in the development of guidelines for accessible learning resources, such as: web pages, videos, textbooks, graphs, and images [5,6]. Efforts have only just begun for creating guidelines for accessible interactive simulations, and the accessibility community does not have the disciplinary pedagogical content knowledge necessary to address this challenge alone. Prior work provides some guidance for making select simple interactive learning environments more accessible, but fails to fully address the challenges that complex environments, such as PhET simulations, present [7].

In this work, we focus on the design and use of accessibility features in a PhET sim (keyboard navigation and auditory descriptions) necessary to support a student with a visual impairment. Additionally, we highlight our efforts to integrate implicit scaffolding within the design of these accessibility features. Note, these accessibility features are intentionally designed to layer onto the existing sim. Rather than creating a new sim specifically for students with certain disabilities, we chose to add accessibility features to the existing sim to support all students in collaborating together, regardless of the presence or absence of a disability.

\section{CAPACITOR LAB: BASICS AND IMPLICIT SCAFFOLDING}

We utilized the unpublished sim Capacitor Lab: Basics (http://goo.gl/SQnzcQ) for this work. This sim can be used to introduce high school and undergraduate students to foundational concepts related to capacitors. The sim's primary learning goals include:

1. Predict how capacitance changes when the plate area or plate separation changes.

2. Describe how charge drains away from a capacitor into a light bulb.

3. Explain the relationships between voltage, charge, stored energy and capacitance.

This sim contains a Capacitance screen and a Light Bulb screen. The Capacitance screen (Fig. 1a) contains a battery and capacitor in a circuit, a capacitance bar graph, a control panel with options for changing what is viewed, and a 


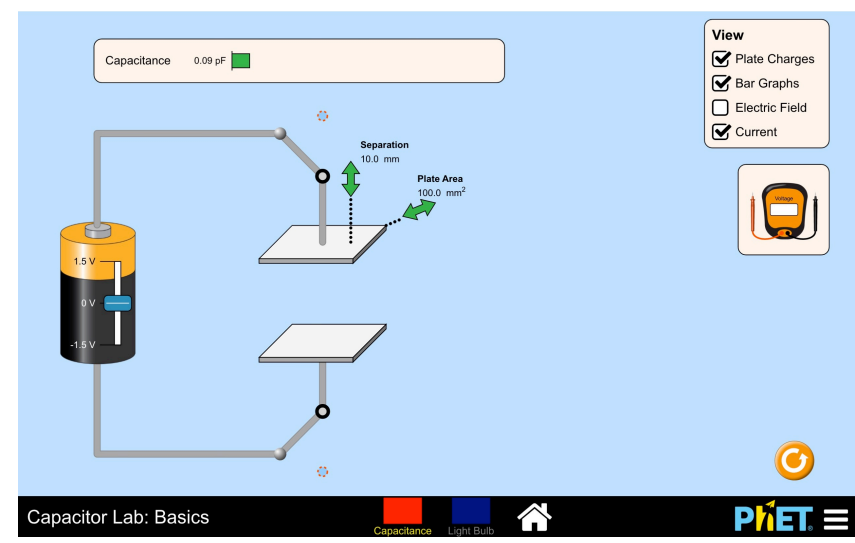

Figure 1a. Screenshot of Capacitance Screen

voltmeter. Students can adjust the plate area and separation and determine how these changes result in a change in capacitance (Learning Goal 1). Students can also change the battery voltage, view plate charge, electric field, and use the voltmeter to determine voltage across different points in the circuit. By interacting with these features, students can learn the relationship between voltage, charge, and capacitance (Learning Goal 3).

The Light Bulb screen (Fig. 1b) introduces a light bulb as a new element in a circuit, as well as options to view bar graphs for plate charge and stored energy. Using this screen, students can explore the relationship between voltage, charge, stored energy, and capacitance (Learning Goal 3). By charging the capacitor and connecting it to the light bulb circuit, students can explore charge behavior between the capacitor and the light bub (Learning Goal 2).

Through the use of implicit scaffolding, these screens have been designed to encourage students to explore and address the sim's learning goals without the use of explicit guidance (e.g., instructions within the sim) [8]. For example, on the Capacitance screen, the prominence of the capacitance graph encourages students to explore what sim interactions impact this graph. In the Light Bulb screen, students recognize that the primary difference between this screen and the Capacitance screen is the addition of the light bulb, and so "turning on the light bulb" often becomes a primary focus.

\section{A. Prototype Accessibility Features}

To increase the accessibility of the Capacitor Lab: Basics sim for students with visual impairments, we designed and implemented two new features for the sim: keyboard navigation and auditory descriptions. A one-minute demo with a brief explanation (https://goo.gl/Swb6N6) of these features is available. Keyboard navigation allows students to navigate and interact with the sim and its representations using solely keyboard input. Auditory descriptions provide verbal descriptions of context (e.g., description of screen), and dynamic feedback (e.g., reading out the change in the

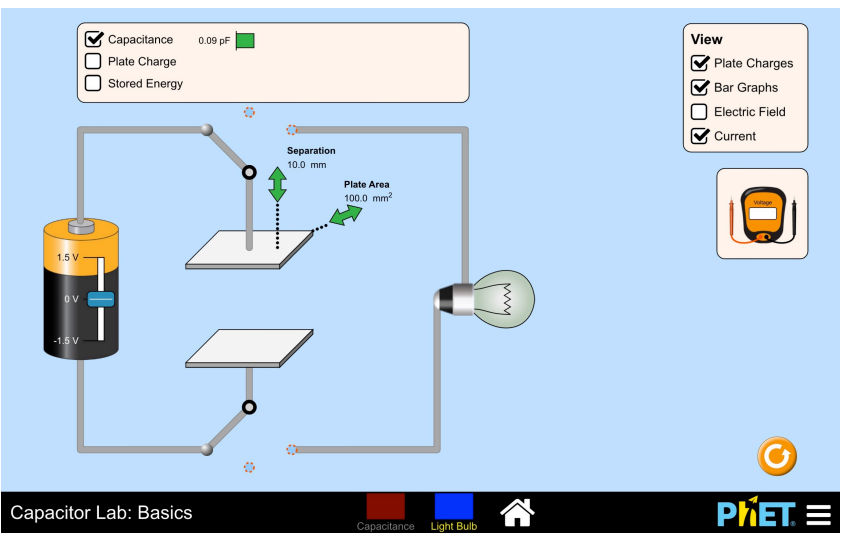

Figure 1b. Screenshot of Light Bulb Screen

capacitance graph when the plate separation is adjusted). These descriptions are verbalized to the student through the use of screen reader software. The act of moving focus around the screen, using their keyboard, and playing with interactive elements, is intended to allow students with disabilities to determine what they can do with the sim and develop conceptual questions to explore [1]. Our goal is to expand our implicit scaffolding design principles beyond the visual, and to ensure that all students are supported in having intuitive and effective learning experiences.

\section{METHODOLOGY}

To investigate how productive the prototype accessibility features were in supporting learning with the sim, we conducted two individual interviews. Each participant described themselves as blind, was an experienced screen reader user, had not taken a physics course since high school, and did not have, or were not, pursuing a degree in a STEM field. Olivia (pseudonym) was a recent college graduate, and Carey (pseudonym) was a current undergraduate student. Data collection included: observation notes; video recordings that captured audio, the computer screen, and the computer keyboard; and screen capture recordings of sim use.

Each interview started with the participant being asked to answer three content questions (pretest). Then, participants used the sim for 20-30 minutes while "thinking aloud." After sim use, participants were read aloud three content questions (posttest). The pre and posttest questions were the same, and designed to probe the participants' understanding of the sim's learning goals. Below is a subset of the questions asked. One question, correlated to Learning Goal 3 , was excluded from final data analysis because both students were unable to answer after incomplete technological implementation prevented them from accessing some necessary information.

- Question 1: In a capacitor, how can you change plate area and plate separation to achieve maximum capacitance? 
- Question 2: What happens when a charged capacitor is connected to a light bulb?

Notes on Sim Prototype Used: These interviews were conducted as part of an iterative design process for developing the accessibility features for Capacitor Lab: Basics. The prototype used for these interviews was fully keyboard accessible, though not all keyboard interactions were optimized. Relevant to the interviews in this study, 1) the keyboard interaction for using the voltmeter was not intuitive for one participant (Carey), 2) the interviewer rather than the screen reader - voiced numerical changes to the capacitance graph for the other participant (Olivia), and 3 ) the plate charge and electric field did not have associated auditory descriptions implemented for either participant causing Question 3 to be eliminated from analysis.

\section{DATA AND RESULTS}

Here, we present analysis of Olivia and Carey's verbalizations, sim interactions, and pre and posttest responses.

\section{A. Olivia}

Olivia's responses to the pretest questions included the following quotes:

- Question 1 Response: "I think closer increases, larger area increases. The further apart you are, the less force between the charges. Well, actually maybe you want to decrease area because increasing area might decrease density. ... The larger battery has more power, like the car battery is much bigger than, you know, a AAA battery."

- Question 2 Response: "Charges go circular."

While these responses from Olivia show that she was actively trying to reason out her responses, they do not provide evidence that Olivia has prior knowledge specific to capacitors. For example, Olivia use language atypical of the topic to describe her ideas, such as "capacitor strengthens" to describe the change to a capacitor upon increasing voltage, and "circular" rather than a more specific reference to a circuit.

After listening to a scene description, and exploring all the sim's interactive features, Olivia began questioning how the interactive features were related - looking for the "point" of the sim. All times are in the form, min:sec, based on the start of sim use.

11:39 "I'm looking for where it would tell me ... so if I can control voltage, I can control size and I can control distance ... so that all goes together to ... so what's the output?"

13:20 Olivia navigates to the capacitance graph, pausing to listen to its description: 'Capacitance graph, measures the capacitance of the capacitor. '...
"I guess I'm looking for the capacitance."

Olivia goes on to spend the majority of her sim use time to investigate Learning Goal 1 - how capacitance is affected by changes to the plate area and separation.

14:25 "I assume that if I push the voltage up the capacitance is also going to go up, but it might stay the same because it might not be dependent on voltage." ... Olivia doubles the voltage ... "Ok, so voltage does not impact [the capacitance]."

15:29 Olivia transitions to adjusting area of capacitor plates. "I had it at 121 ... so if I go up ..." Olivia hears that capacitance is higher ...

18:01 "I'm going to take the size back down to where the default was..." Olivia then decreases the plate separation, pausing to hear the change in capacitance.

Olivia's conclusion that it would be useful to explore the effect of various sim features on capacitance, without any explicit instructions, indicates that the auditory descriptions provided implicit scaffolding that cued her to engage in these productive investigations. Olivia spent the entirety of her sim interaction on the Capacitance screen, and did not use the Light Bulb screen.

Olivia's responses to the posttest questions included the following quotes:

- Question 1 Response: "[Separation of plates] Closer together and larger [plate area]."

- Question 2 Response: "The electrons flow through the circuit and go to the light bulb."

In her response to the first learning goal, Olivia appeared to gain confidence, compared to her pretest responses, in which she struggled as to whether or not increasing area would increase capacitance. The original learning goal only asks to predict how capacitance changes, and therefore Olivia's confidence in this answer suggests that she was able to master this learning goal while using the sim.

For the second learning goal, Olivia's response indicates that she successfully learned the concept in the context of the language of the sim. Her use of electrons, flow, circuit, and light bulb; suggest that she successfully gained a mental image of how the capacitor and light bulb connect via the circuit. This is significant because even though she never accessed the Light Bulb screen, Olivia was still able to understand how it would operate based on her exploration of the Capacitance screen.

\section{B. Carey}

Carey's responses to the pretest questions included the following quotes:

- Question 1 Response: "I have no idea."

- Question 2 Response: "It turns the light bulb on. Isn't a capacitor like a battery, or some sort of power source?" 
Our interpretation of these responses is that Carey has some association with a capacitor as an energy source but lacks, or is unable to utilize, any further prior knowledge to help her engage in reasoning around the question topics.

After navigating through all the sim's interactive features, Carey found the implementation of the voltmeter to be unintuitive, and subsequently spent a significant amount of time attempting to place the voltmeter probes on the circuit. Her persistence in trying to understand how to use the voltmeter limited the amount of productive time she had with the sim.

After 16 total minutes of navigating the sim in attempt to interact with the voltmeter, the interviewer informed Carey of the option to switch to the Light Bulb screen. Without further prompting, Carey immediately noted the addition of the light bulb, and focused on determining how to turn it on. She was able to accomplish lighting the light bulb in about two minutes.

27:02 Carey connects the capacitor to the battery, and then connects the capacitor to the light bulb. "Hooray the light bulb lit!"

Through the presence of the light bulb and intuitive keyboard interactions for the battery, capacitor, and circuit switches, Carey was able to target a productive goal in the sim and quickly experience success.

Carey's responses to the posttest questions included the quotes below:

- Question 1 Response: "Increase the [plate] area and decrease the distance between them ... that's just something that sounds good"

- Question 2 Response: "It turns the light bulb on."

After use of the sim, Carey is able to engage in reasoning, though tentatively, around how to increase capacitance, even though she had no prior knowledge, or even ideas, about how to change capacitance.

\section{CONCLUSIONS}

The round of interviews described in this paper is the first of many in an effort to make Capacitor Lab: Basics accessible. The results described in this paper are promising, showing that well-designed keyboard navigation with auditory descriptions can support a range of interactions and the learning of foundational physics concepts. In the interviews, we see that both users are prompted by descriptions of an element in the sim. Even though Olivia spends significant time collecting and analyzing data on the capacitance graph, while Carey accomplishes the satisfaction of lighting the light bulb, each achieve a Learning Goal in the process. Both Olivia and Carey's gains in confidence on the topic of changing capacitance, and their increased use of sim specific vocabulary, show that they were able to make noticeable learning gains. These results support the implementation of accessibility features in interactive learning resources, such as PhET, for the use of blind learners. Additionally, this work shows that implicit scaffolding can be successfully integrated into auditory descriptions to engage users in productive interactions, without explicitly telling them what steps to take.

While we were able to make substantial gains in the implementation of accessibility features, there is much remaining work to be done in the effort to make PhET Interactive Simulations completely accessible for all users. Both Olivia and Carey struggled due to various unimplemented or unrefined features, showing that challenges with the sim can derail the learning experience. It is important to have well designed and implemented accessibility features to support intuitive interactions and minimize distraction from the exploring and learning process. To learn more about PhET's efforts in accessibility, visit http://phet.colorado.edu/en/accessibility.

\section{ACKNOWLEDGEMENTS}

I would like to thank Jesse Greenberg and Emily Randall for their work in implementation of the accessibility features of Capacitor Lab: Basics, as well as the contributions of Emily Randall's undergraduate thesis [9] to this research. Funding for this work was provided by the National Science Foundation (DRL \#1503439), the University of Colorado Boulder, and the William and Flora Hewlett Foundation.

\section{REFERENCES}

[1] E. B. Moore and T. L. Smith. Universal Access in Human-Computer Interaction. In Press (to be published mid-July).

[2] NAEP. The Nation's Report Card: Science 2011 (NCES 2012-465).

[3] http://www.nationsreportcard.gov/science 2011/g 8 nat.aspx?subtab $\mathrm{id}=\mathrm{Tab} 6 \& \mathrm{tab} \mathrm{id}=\mathrm{tab} 1$ - chart. Retrieved 6/22/16.

[4] http://education.wm.edu/centers/ttac/resources/arti cles/inclusion/cooperativelearning/index.php Retrieved 6/16/16.

[5] http://www.washington.edu/doit/accessiblescience-equipment Retrieved 6/16/16.

[6] http://www.washington.edu/doit/resources/popular -resource-collections/accessible-technology Retrieved 6/02/16.

[7] https://www.imsglobal.org/accessibility/accessible vers/sec8.html Retrieved 6/22/16.

[8] N. F. Podolefsky, E. B. Moore, and K. K. Perkins. arXiv: 13066544.2014

[9] E. Randall. Undergraduate Honors Thesis, University of Colorado Boulder, 2016. 